\title{
Generation of Double Pulses in the Extreme Ultraviolet Spectral Range Using a Laser Combined Pinch Plasma Source
}

\author{
S. Brückner ${ }^{1}$, S. Wieneke ${ }^{2}$ and W. Viöl ${ }^{2, *}$ \\ ${ }^{I}$ University of Applied Sciences and Arts, Von-Ossietzky-Str.99, D-37085 Göttingen, Germany \\ ${ }^{2}$ Laser-Laboratorium Göttingen e.V. (LLG), Hans-Adolf-Krebs-Weg 1, D-37077 Göttingen, Germany
}

\begin{abstract}
The interaction of a dense plasma with short laser pulses (ns) is investigated. The dense plasma is created using a low-current hollow-cathode-triggered discharge. This configuration generates a dense plasma with an electron density of $n_{e} \approx 10^{17} \mathrm{~cm}^{-3}$. Spectra are taken in the extreme ultraviolet (EUV) spectral range from $11-18 \mathrm{~nm}$ to estimate the temperature. The interaction between plasma and laser beam as a longitudinally pumped light source is discussed. The required properties of the plasma, according to the radial profiles of the temperature and the density, are exemplified. It is shown, that the generation of double pulses in the extrem ultraviolet spectral range is possible by reheating the residuals of an expanding dense plasma with a short laser pulse.
\end{abstract}

Keywords: Laser heating, inverse bremsstrahlung, plasma heating, z-pinch, hollow cathode.

Soft X-ray radiation in the range from $2-13 \mathrm{~nm}$ is of great interest for the micro- and nano-structuring, as well as for the structural analysis. Further, the special characteristics of the light-matter interaction in this spectral region provide a very accurate mean for elemental and chemical speciation [1]. Today, for the generation of such a radiation, two commercial techniques are established. On the one hand there are gas discharge-based sources and on the other hand there are laser produced plasmas. High efficiencies and simple source designs are provided by the gas discharge techniques [2]. A major disadvantage of gas discharge-based EUV-sources are the large currents (20-50 kA [3]) and thus, the reduced lifetime of the optical elements as well as the lifetime of the source it self. In laser produced plasmas (LPP), the generation of debris can be minimized when the whole mass of the target is converted into plasma entirely. Such mass-limited targets can be a liquid jet, small droplets or atomic clusters [4-6]. Disadvantages of LPP systems are the high complexity of the target generation and the relatively high expenses for the according laser systems.

The recent development according to hybrid excitation is mainly aimed at the soft X-ray laser research. Hybridly excited capillary discharges are examined as a compact $\mathrm{X}$ ray laser source at various facilities at present [7-10]. In this case, a compressed plasma column is additionally heated using ps-laser pulses to obtain population in version during the phase of the subsequent de-excitation and recombination. In some facilities, however, the heating effect of ns-laser pulses on plasma plumes is under investigation to examine the effect of such a laser heating with respect to the (incoherent) EUV yield and the conversion efficiency [1113].

*Address correspondence to this author at the Laser-Laboratorium Göttingen e.V. (LLG), Hans-Adolf-Krebs-Weg 1, D-37077 Göttingen, Germany; Tel: 00495513705 218; Fax: 00495513705 206; E-mail: wolfgang.vioel@llg-ev.de
This paper deals with the combination of a gas discharge in the special form of a hollow cathode-triggered (HCT) discharge with short laser pulses for the generation of two time-shifted EUV radiation pulses. This approach unites essential advantages of two well-established techniques and minimizes at the same time their disadvantages. Such a soft $\mathrm{X}$-ray/EUV source can form the base for an efficient, compact and cost-efficient radiation source for lots of laboratory applications, e.g. for pump-probe experiments to obtain information on ultra fast phenomena.

\section{EXPERIMENTAL BACKGROUND}

The schematic representation of the experimental setup is shown in Fig. (1). A capacitor bank $(46 \mathrm{nF}, 9.2 \mathrm{nH})$ is charged with up to $7 \mathrm{kV}$ which corresponds to $1.1 \mathrm{~J}$ of electrical energy, and serves as a power supply to the discharge. The electrode system is a hollow cathodetriggered (HCT) z-pinch design. Further details concerning such a device can be found in several publications [3, 14]. When the discharge is triggered, an electrical breakdown follows with a rate of current rise reaching as high as $\approx 10^{11}$ $\mathrm{A} / \mathrm{s}$. The resulting current duration is about $200 \mathrm{~ns}$ (typical discharge durations are range from 100 to $500 \mathrm{~ns}$ [15]). A pinch plasma is formed due to the magnetic selfcompression, which originates from the fast current rise. Since the bore hole diameters are relatively small $(\mathrm{d}=4 \mathrm{~mm})$, the minimum plasma diameter is reached already at $t \approx 40 \mathrm{~ns}$ after the beginning of the break down $(t=0)$. The period of peak compression has a duration of about $15 \mathrm{~ns}$ and within this time the electron density amounts $n_{e} \approx 10^{17} . .10^{18} \mathrm{~cm}^{-3}[16$, 17]. Then the plasma expands and cools down due to pressure reduction, further radiative losses and heat conduction. Of course is this phase of the discharge also characterized by a quick de-excitation and recombination of the previously highly ionized species. Those residuals are to be reheated by a short laser pulse. In order to give more or less an idea of the actual heating effect, i.e. the difference of 


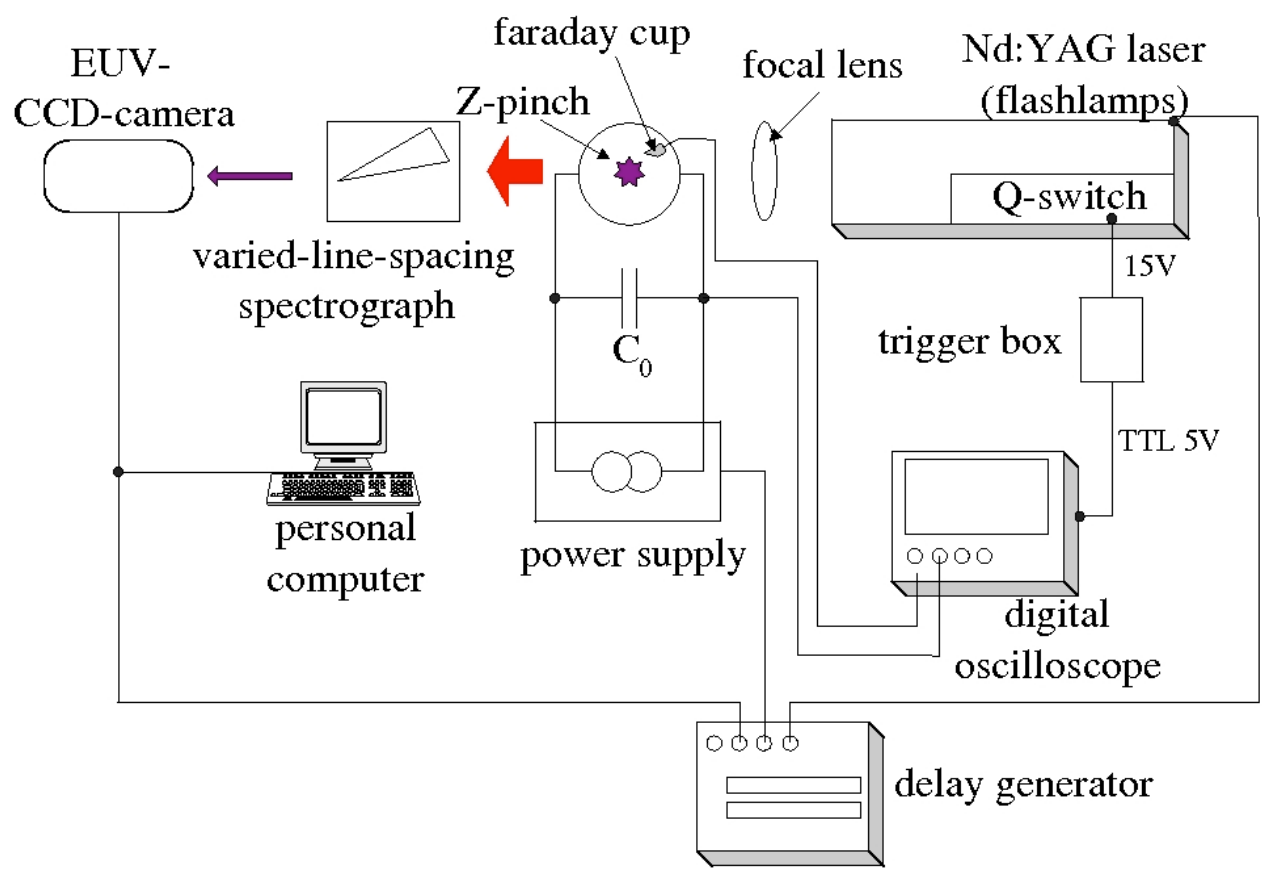

Fig. (1). Schematic representation of the experimental setup to synchronize the z-pinch discharge with the laser pulse.

the electron temperature before and during the laser pulse, simulations were carried out (see Part 4).

A focal lens is used to focus the laser pulse on the axis of rotation of the z-pinch plasma. The used Nd:YAG laser system delivers pulses with a duration of $9 \mathrm{~ns}$ (fwhm) and an energy of up to $800 \mathrm{~mJ}$. Due to the unavoidable delays caused by the electronics itself and several meters of coaxial cable, no suitable trigger signal can be obtained from the voltage or current characteristics early enough to trigger the q-switch of the laser. Therefore a faraday cup-like detector is utilized in order to synchronize the laser with the pinch plasma. The typical geometrical design of the hollowcathode leads to an axial electron beam (dark current) just before the main breakdown occurs. This arrangement delivers a sufficient strong signal prior to the electrical breakdown of the hollow cathode. The delay between this trigger signal and the breakdown mainly depends on the working gas. It roughly amounts $80 \mathrm{~ns}$ for xenon and $100 \mathrm{~ns}$ for argon. Since the laser needs about $220 \mathrm{~ns}$ from triggering to the emission of the laser pulse the plasma cannot be hit in pinched state $(\approx$ $40 \mathrm{~ns}$ after breakdown). In the present setup, the reachable minimum delay between pinch time and laser pulse is therefore limited to $\Delta \mathrm{t} \approx 80 \mathrm{~ns}$ for argon and $\Delta \mathrm{t} \approx 100 \mathrm{~ns}$ for xenon. However, the decay of the plasma is slow enough to be heated up a second time.

\section{ABSORPTION COEFFICIENT}

The absorption of laser radiation in a plasma, and thus the heating-up of the plasma, is affected by the inverse bremsstrahlung in the regime considered here. In this process a free electron accepts a radiation quantum of the laser beam. For reasons of the impulse conservation this is only possible in interaction with a neutral atom or an ion. The atoms or ions only absorb a small amount of the radiation energy and are primarily excited and heated indirectly by collisions with the accelerated electrons. While primarily neutral atoms exist at the beginning of the ignition, the electron-atom inverse bremsstrahlung dominates at that time. The according absorption coefficient $\alpha_{\text {ea }}$ is given as

$\alpha_{e a}=\frac{1}{4 \pi \varepsilon_{0}} \cdot \frac{e^{2} v_{e a} n_{e} \lambda_{L}^{2}}{\pi m_{e} c^{3}}$.

Here $n_{e}$ is the electron density, $\lambda_{L}$ the laser wavelength and $v_{e a}$ is the impact frequency for impulse-exchanging collisions between electrons and neutral atoms. In an already ignited discharge, however, the electron-ion inverse bremsstrahlung plays the decisive role for the heating-up of the plasma. The absorption coefficient $\alpha_{e i}$ for electron-ion inverse bremsstrahlung applies to

$\alpha_{e i}=\frac{n_{e}^{2} e^{6}}{6 \sqrt{3} n \varepsilon_{0}^{3} c \hbar \omega_{L}^{3} m_{e}^{2}} \cdot \sqrt{\frac{m_{e}}{2 \pi k_{B} T_{e}}} \cdot\left(1-e^{-\frac{\hbar \omega_{L}}{k_{B} T_{e}}}\right) \bar{g}$

where $\omega_{L}$ is the laser frequency and $n$ is the real part of the refractive index. The averaged Gaunt-factor $\bar{g}$, as adjustment to relativistic and quantum mechanical effects, applies to:

$\bar{g}=\frac{\sqrt{3}}{\pi} \sqrt{\frac{\pi k_{B} T_{e}}{\hbar \omega_{L}}}$.

In this paper, the Nd:YAG laser is considered. In that case, a more precise expression for the Gaunt-factors can be written as:

$\bar{g}_{N d: Y A G}=6.2 \times 10^{-3} \cdot\left(\frac{T_{e}}{K}\right)^{0.42}+0.86$.

Fig. (2) shows the linear absorption coefficient as a function of the plasma temperature for different electron 


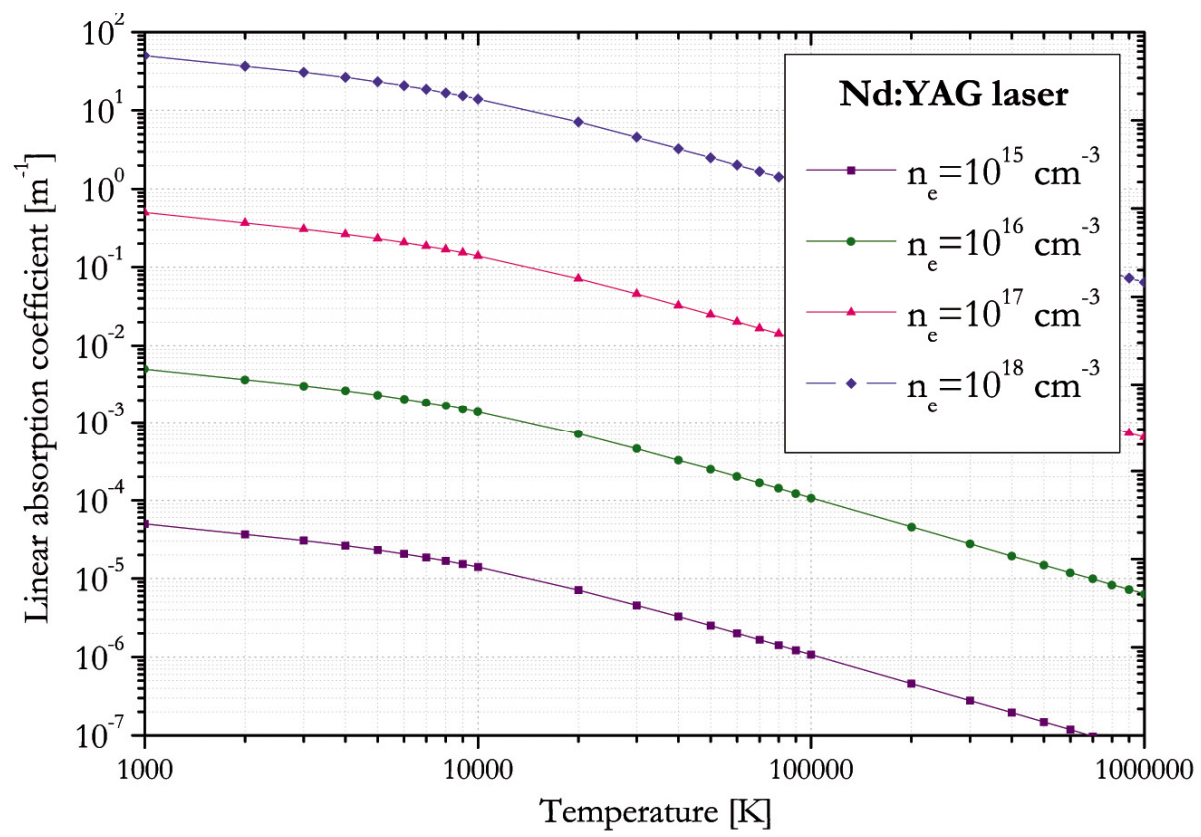

Fig. (2). Linear absorption coefficient for a Nd:YAG laser with a wavelength of $1.06 \mu \mathrm{m}$ as a function of the electron temperature for different electron densities.

densities $n_{e}$. These graphs clarify that, in order to achieve an effective absorption of laser radiation, the plasma requires a high electron density and a low electron temperature. Janulewicz et al. found that the absorption medium demands a concave electron density distribution with the maximum on the axis, against what the temperature distribution is to behave in reverse [8]. This distribution is fulfilled in z-pinch plasmas and is experimentally determined in [17].

\section{EXPERIMENTAL RESULTS}

Fig. (3) shows the experimentally determined extreme ultraviolet spectra of a xenon z-pinch plasma combined with a short laser pulse. The time difference between the z-pinch plasma (in pinched state) and the incident laser pulse is about100 ns. As a consequence the laser does not hit the plasma at peak compression but rather the residuals of the discharge. As shown the intensity over the whole spectral range increases at about a factor of two compared to the sole z-pinch plasma, whereas the sole laser without a discharge has no effect (green line). The spectra are taken from a single shot each. A comparison of the spectral line intensities of each spectra, as shown in [17], leads to an estimated electron temperature of $\mathrm{T}_{e} \approx 70 \mathrm{eV}$ in both cases. The same experiment was carried out using argon as absorption

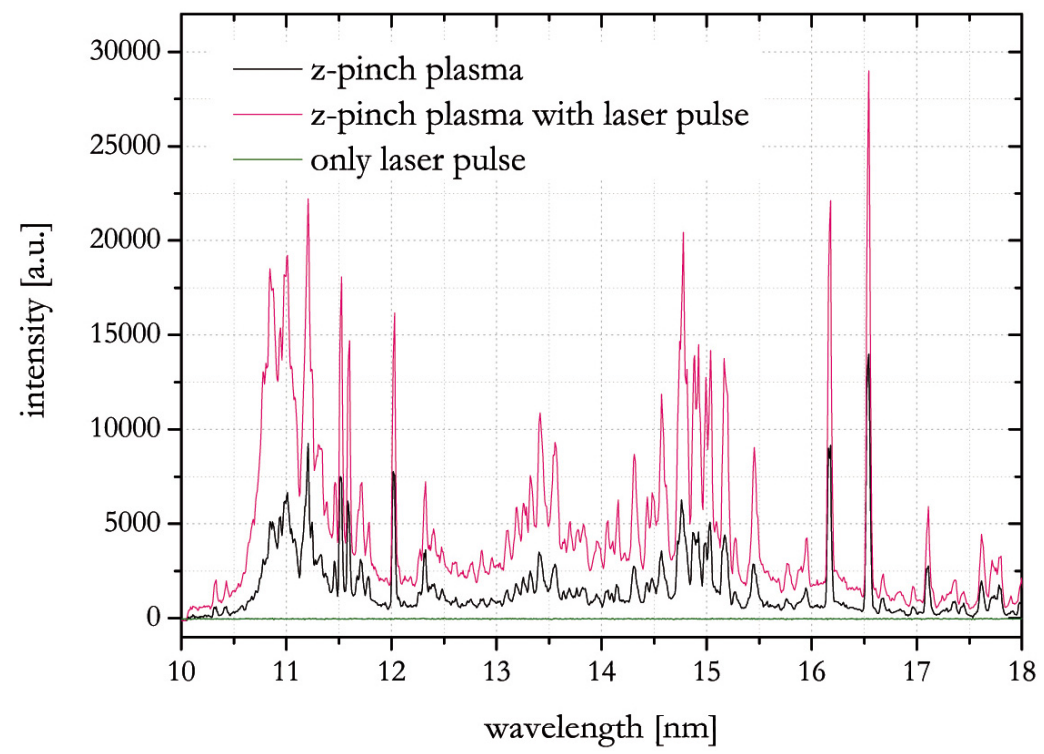

Fig. (3). Experimentally determained EUV spectra of a xenon z-pinch plasma (black) and a combined laser pulse reheated zpinch plasma (red). In comparison the sole laser pulse does not create any EUV radiation, because the charge carrier density is to low for the ignition of a plasma discharge (green). 




Fig. (4). Comparison of experimentally determained EUV spectra of an argon z-pinch plasma spectra with an initial pressure of 6.5Pa with (red) and without (black) an additional laser pulse heating.

medium. In this case the time difference between the z-pinch plasma and the incident laser pulse is about $80 \mathrm{~ns}$ and the intensity approximately increased by a factor of 3 (see Fig. (4)). In this experiment it is also apparent that there is only an increase in the intensity but no change in temperature. The electron temperature can be estimated to be $\mathrm{T}_{e} \approx 27 \mathrm{eV}$.

\section{SIMULATION OF LASER COMBINED PINCH PLASMAS}

The time-shift between the generation of the pinch plasma and the laser pulse plays the key role for the efficient combination. This assumption is examined with the help of some simulations more precisely using Helios. Due to the limited possibilities of simulating laser and pinch-plasma combinations with Helios some simplifications and assumptions are made. The basic idea is to describe the pinch plasma as a (laser induced) prepulse with a specific energy and a certain full width of half maximum. This prepulse is used to generate the plasma. A second main pulse is used to heat up or to reheat the plasma. Fig. (5) shows the distribution of the double pulse used for the simulations.

\section{MATHEMATICAL MODEL}

To compare the experimentally determined electron temperature with model calculations the HELIOS code was used. HELIOS-CR is a user-oriented 1-D radiation

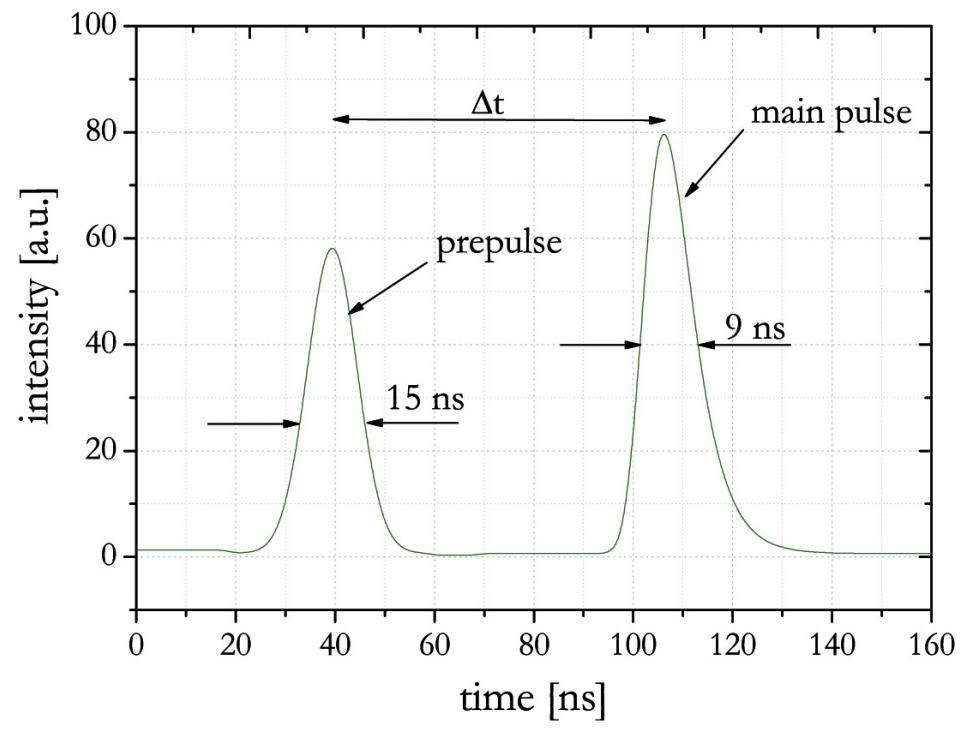

Fig. (5). Sample of the double pulse characteristics used for the simulations. In the simulations the temporal distance between pre-and main pulse is set to $\Delta t=0 \mathrm{~ns}, \Delta t=20 \mathrm{~ns}, \Delta t=100 \mathrm{~ns}$. 

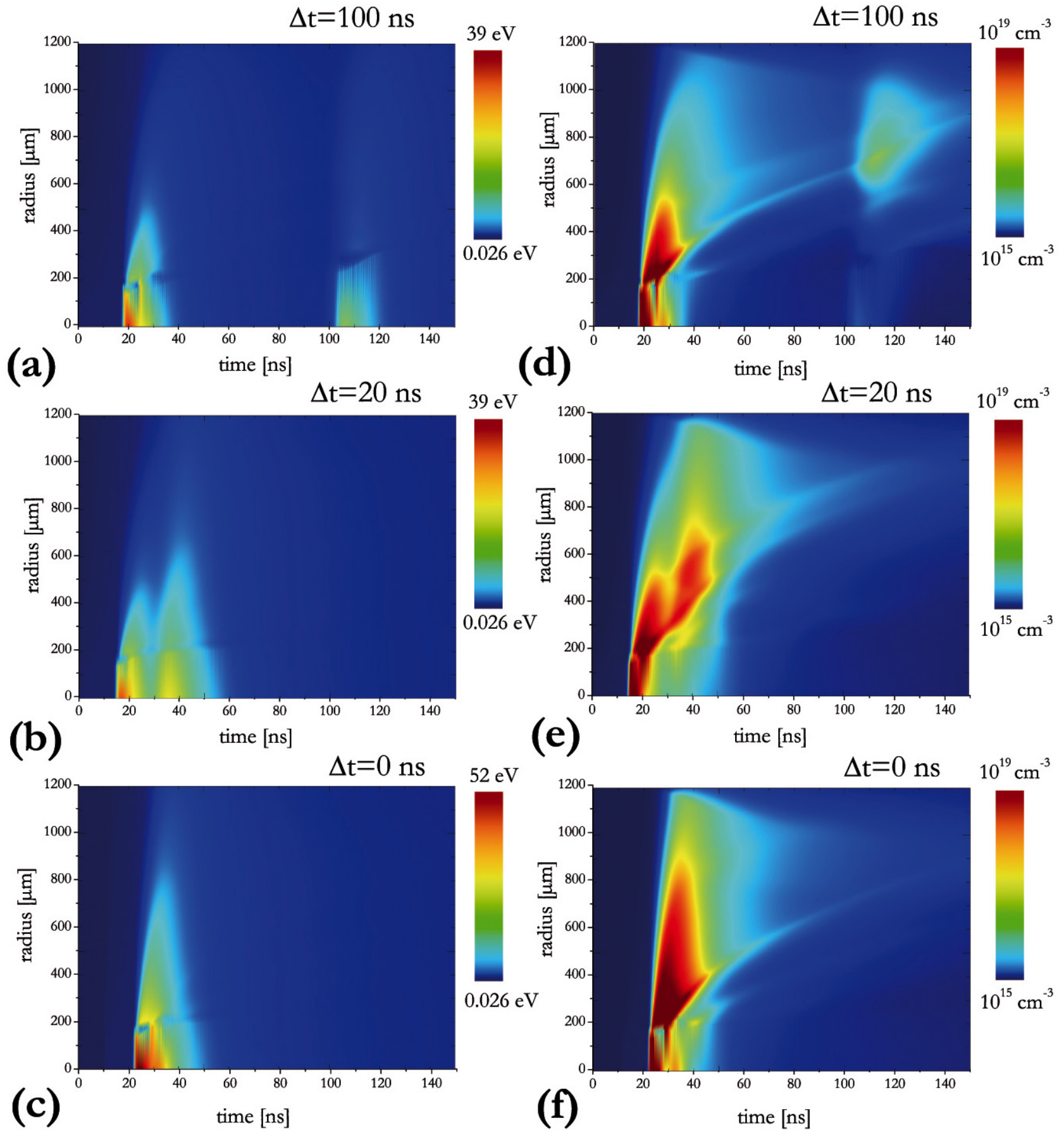

(e) time $[\mathrm{ns}]$

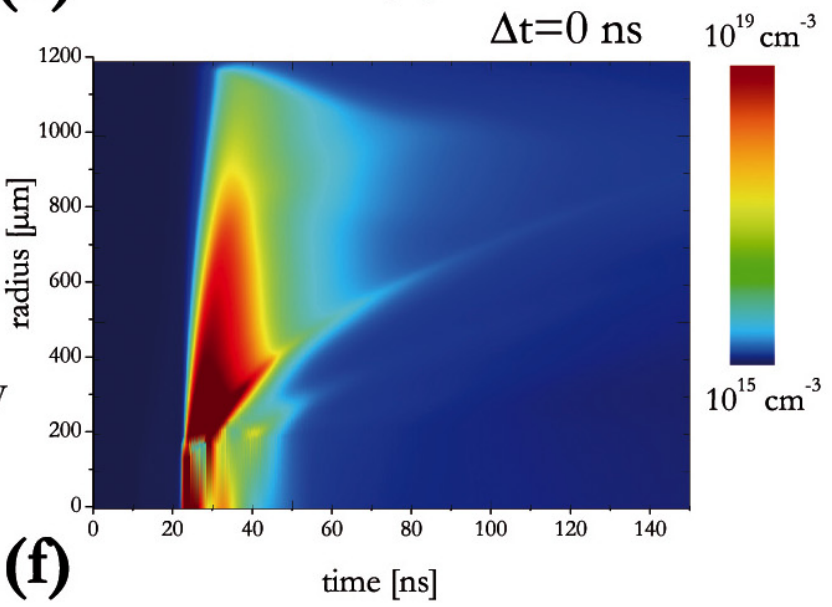

Fig. (6). Plot of the simulated electron temperature (a-c) and the electron density (d-f) for different delays. The gas is xenon at a pressure of 6.5 Pa. It is shown that the residuals of the pre-pulse can be reheated. An additional heating of the z-pinch plasma occurs, if the delay is set to $\Delta t=0 \mathrm{~ns}$.

magnetohydrodynamics code to simulate the dynamic evolution of plasmas created in high energy density physics (HEDP) experiments, i.e. z-pinch plasmas. HELIOS-CR supports the utilization of equation of state and opacity databases that are generated under the assumption of local thermodynamic equilibrium (LTE), as well as those generated for non-LTE plasmas. It includes an in-line collisional radiative (CR) model for computing non-LTE atomic level populations at each time step of the hydrodynamics simulation. The energy equations employed include models for laser energy deposition, radiation from external sources, and high-current discharges. Radiative transport can be calculated using either a multi-frequency flux-limited diffusion model, or a multi-frequency, multiangle short characteristics model. HELIOS-CR supports the use of SESAME equation of state (EOS) tables, PROPACEOS EOS/multi-group opacity data tables, and non-LTE plasma properties computed using the inline CR modeling. HELIOS-CR solves Lagrangian hydrodynamics equations in planar, cylindrical, and spherical geometries. 


\subsection{Laser Energy Deposition}

The laser deposition model in HELIOS-CR utilizes ray tracing algorithms. It is assumed that laser light propagates through the plasma instantaneously. Effects due to the polarization of laser light are currently neglected. Laser energy is deposited in the plasma using an inverse bremsstrahlung model when the electron density is less than the critical density. Laser energy is not allowed to penetrate beyond the critical surface. The depth at which the laser light penetrates is determined from the absorption coefficient.

\subsection{Mass Conservation Equations}

In a Lagrangian hydrodynamic system, the mass of each volume element is conserved because the spatial grid moves with the mass. For a cylindrical plasma the mass of each volume element is given by

$d m_{0}=\rho(r) r d r$,

where $\rho$ is the mass density, $r$ is the spatial position (i.e., the radius in cylindrical geometries). In this system, the continuity (mass conservation) equation is automatically satisfied. The momentum conservation equation is solved by the one-fluid approximation, where the plasma electrons and ions are assumed to flow together as a single fluid. The momentum conservation equation for a cylindrical geometry is given by:

$\frac{d u}{d t}=-r \frac{\partial}{\partial m_{0}}(p+v)-\frac{1}{8 \pi r} \frac{\partial}{\partial m_{0}}\left(r^{2} B^{2}\right)$,

where $u$ is the fluid velocity, $t$ is the time, $p$ is the total pressure due to electrons, ions, and radiation, $v$ is the Von Neumann artificial viscosity, and $B$ is the magnetic field induced by the axial current. The Von Neumann artificial viscosity term is included to effectively smooth shocks by spreading out a rapid increase in pressure over a small number of zones [18]. To simulate z-pinch plasmas a magnetic diffusion model must be used.

\subsection{Energy Conservation}

The equations of energy conservation written in terms of temperature diffusion equations are given by

$C_{v, e} \frac{\partial T}{\partial t}=\frac{\partial}{\partial m}\left(r \xi_{e} \frac{\partial T}{\partial r}\right)-\left(\frac{\partial E_{e}}{\partial V}+P_{e}\right) \frac{\partial V}{\partial t}+R_{h}-R_{c}$

and

$C_{v, i} \frac{\partial T}{\partial t}=\frac{\partial}{\partial m}\left(r \xi_{i} \frac{\partial T}{\partial r}\right)-\left(\frac{\partial E_{i}}{\partial V}+P_{i}\right) \frac{\partial V}{\partial t}-v \frac{\partial V}{\partial T}$

where $T$ is the temperature, $C_{v}$ is the specific heat capacity, $\xi$ is the thermal conductivity, $E$ is the specific internal energy, $R_{h}$ is the radiative heating, and $R_{c}$ is the radiative cooling. This equation simply states that specific change in energy rate is the sum of all of the heating and cooling processes. HELIOS solves this series of partial differential equations in order to calculate the time dependent temperature and density distributions. For the Simulations a static xenon environment at a pressure of $6.5 \mathrm{~Pa}$ is chosen. Figs $(\mathbf{5 , 6})$ show the double pulse characteristics used for the simulation and, respectively, the result of the simulation. Both, the double pulse characteristics and the initial gas conditions are geared to the experimental conditions. It is shown that the residuals of the pre-pulse can be reheated significantly.

\section{CONCLUSION AND OUTLOOK}

The interaction between a laser pulse and a z-pinch plasma was investigated. A dense plasma was created using a low-current hollow-cathode-triggered discharge. Spectra were taken in the extreme ultraviolet (EUV) spectral range from 11-18 nm to estimate the temperature. The interaction between plasma and laser beam as a longitudinally pumped light source is discussed. It is shown, that the generation of double pulses in the extreme ultraviolet spectral range is possible by reheating the residuals of an expanding dense plasma using a short laser pulse. As a result the radiation yield of a xenon z-pinch discharge was increased by a factor of 2 and the radiation yield of an argon z-pinch discharge was increased by a factor of 3 , respectively. Our future work will concentrate on making the delay tunable and working gas independent. Then it should be possible to align the pinch plasma and the laser pulse in order to reheat the plasma in different states. This leads to specific emission characteristics for different applications. There is also a large demand to improve the simulations in order to describe the actual experimental situation more precisely.

\section{ACKNOWLEDGMENT}

This project is sponsored by the European Fonds for Development (EFRE) and the Workgroup Innovative Projects of Lower Saxony of Germany(AGIP) to whom we want to express our special gratitude.

\section{REFERENCES}

[1] Attwood D. Soft X-Rays and Extreme Ultraviolet Radiation: Principles and Applications. Cambridge University Press 1999.

[2] Stamm U, Schwoerer H, Lebert R. Strahlungsquellen für die EUVLithographie. Phys J 2002; 1(12): 33-9.

[3] Bergmann K, Rosier O, Lebert R, Neff W, Poprawe R. Multikilohertz pinch plasma radiation source for extreme ultraviolet lithography. Microelectron Eng 2001; 57-58: 71-7.

[4] Vogt U. Röntgenemission aus laserinduzierten Plasmen: Einfluss von Laserintensität und Pulsdauer bei verschiedenen Targetsystemen. Ph.D. diss., Fakultät Mathematik und Naturwissenschaften, Technische Universität Berlin 2002.

[5] Son J, Cho M, Kim D, Ahn B, Kim J. Prepulse effect on laserinduced water-window radiation from a liquid nitrogen jet. Appl Phys Lett 2007; 90: 261502.

[6] Peth C, Kalinin A, Barkusky F, Mann K, Toennies JP, Rusin LY, XUV Laser-plasma source based on solid Ar filament. Rev Sci Instrum 2007; 78: 103509

[7] Heinbuch S, Grisham M, Martz D, Rocca JJ. Demonstration of a desk-top size high repetition rate soft $\mathrm{x}$-ray laser. Opt Express 2005; 13: 4050-5.

[8] Janulewicz KA, Lucianetti A, Priebe G, Nickles PV. Review of state-of-the-art and output characteristics of table-top soft X-ray lasers. X-Ray Spectrom 2004; 33: 262-6.

[9] Janulewicz KA, Rocca JJ, Bortolotto F, et al. Demonstration of a hybrid collisional soft-X-ray laser. Phys Rev A 2001; 63: 033803

[10] Daido H. Review of soft X-ray laser researches and developments. Rep Prog Phys 2005; 65: 1513-76.

[11] Tao Y, Tillack MS, Harilal SS, Sequoia KL, Najmabadi F. Investigation of the interaction of a laser pulse with a preformed Gaussian Sn plume for an extreme ultraviolet lithography source. J Appl Phys 2007; 101: 023305.

[12] Fujioka S, Shimomura M, Shimada Y, et al. Pure-tin micro droplets irradiated with double laser pulses for efficient and minimum-mass 
extreme-ultraviolet light source production. Appl Phys Lett 2008; 92: 241502 .

[13] Wieneke S, Brückner S, Viöl W. Laser assisted heating of extreme ultraviolet-emitting z-pinch plasmas. Phys Plasmas 2008; 15: 122508 .

[14] Brückner S, Wieneke S, Viöl W. Theoretical and experimental investigations of the suitability of low-current z-pinch plasma as an absorption medium for laser radiation. Contrib Plasma Phys 2008; 48: $577-85$.
Krücken T, Bergmann K, Juschkin L, Lebert R. Fundamentals and limits for the EUV emission of pinch plasma sources for EUV lithography. J Phys D: Appl Phys 2004; 37: 3213-24. Highly repetitive, extreme ultraviolet radiation source based on a gas discharge plasma. Appl Opt 1999; 38, 5413-7.

[17] Wieneke S, Brückner S, Viöl W. Spectroscopic studies of a hollowcathode-triggered z-pinch discharge. Plasma Sci IEEE Trans 2007; 35(3): 601-5.

[18] von Neumann J, Richtmyer R. A method for the numerical calculation of hydrodynamic shocks. J Appl Phys 1950; 21: 232-7.

Received: December 22, 2008

(C) Brückner et al.; Licensee Bentham Open.

This is an open access article licensed under the terms of the Creative Commons Attribution Non-Commercial License (http://creativecommons.org/licenses/by$\mathrm{nc} / 3.0 /$ ), which permits unrestricted, non-commercial use, distribution and reproduction in any medium, provided the work is properly cited. 